\title{
Towards state estimation of Persidskii systems
}

\author{
Wenjie Mei, Denis Efimov, Rosane Ushirobira *广
}

\begin{abstract}
A state estimation scheme for a class of Persidskii systems is introduced in this paper. Two distinct sets of conditions are formulated for robust stability and convergence of the state estimation error using the theories of input-to-output stability (IOS) and input-to-state stability (ISS). These conditions for the nonlinear error dynamics are established in the form of linear matrix inequalities. Two numerical examples are presented to illustrate the effectiveness of the proposed results.
\end{abstract}

\section{Introduction}

The estimation of unmeasured states is an important problem, that must be solved to better control dynamical systems [1, 2]. It has been extensively investigated in various fields, and numerous solutions were proposed for linear plants (e.g. the most common approaches are Kalman filter [3] or Luenberger observer [4]) or for plants whose models are close to linear under certain restrictions $[5,6,7]$. By applying appropriate first order approximations, main linear approaches for estimation can be developed to nonlinear plants including the extended Kalman filter [8] or moving horizon estimation [9], to mention a few popular techniques. However, if the estimation is required for large deviations of the states, then linearization methods lose their validity and nonlinear dynamical models come to the focus.

The main difficulty in designing nonlinear state estimators consists in the complexity of the stability analysis for the state estimation error (the main approach for the stability analysis of nonlinear dynamics is the Lyapunov function method [1], and there is no constructive tool for selecting a Lyapunov function in the

${ }^{*}$ This work was partially supported by the China Scholarship Council (CSC) Scholarship (CSC no. 201908050104), by the Government of Russian Federation (Grant 08-08) and by the Ministry of Science and Higher Education of Russian Federation, passport of goszadanie no. 2019-0898.

${ }^{\dagger}$ Wenjie Mei, Denis Efimov, and Rosane Ushirobira are with Inria, Univ. Lille, CNRS, UMR 9189 - CRIStAL, F-59000 Lille, France $\{$ Wenjie.Mei, Denis.Efimov, Rosane. Ushirobira \} einria.fr. D. Efimov is with ITMO University, 49 av. Kronverkskiy, 197101 Saint Petersburg, Russia. generic nonlinear case). Another obstacle, hidden but still important, is the estimation error representation: usually, it is assumed that the estimation error dynamics can be presented as an autonomous system (governed by exogenous disturbances and measurement noises), which can be a restrictive hypothesis. To illustrate this issue consider a nonlinear dynamical system:

$$
\dot{x}(t)=f(x(t), d(t)), y(t)=h(x(t)),
$$

where $x(t) \in \mathbb{R}^{n}, d(t) \in \mathbb{R}^{m}$ and $y(t) \in \mathbb{R}^{p}$ are the state, the input and the measured output, respectively, $f: \mathbb{R}^{n+m} \rightarrow \mathbb{R}^{n}$ and $h: \mathbb{R}^{n} \rightarrow \mathbb{R}^{p}$ are some known nonlinear functions. In general, an observer for this system takes the form

$$
\dot{z}(t)=r(z(t), y(t)), \hat{x}(t)=g(z(t)),
$$

where $z(t) \in \mathbb{R}^{q}$ is the state of the observer and $\hat{x}(t) \in \mathbb{R}^{n}$ is the estimate of $x(t), r: \mathbb{R}^{q+p} \rightarrow \mathbb{R}^{q}$ and $g: \mathbb{R}^{q} \rightarrow \mathbb{R}^{n}$ are functions to be selected in a way that the estimation error $e(t)=x(t)-\hat{x}(t)$ dynamics is asymptotically stable for $d=0$ (i.e. $\lim _{t \rightarrow+\infty}\|x(t)-\hat{x}(t)\|=0$ ) and robustly stable in the presence of disturbances $d \neq 0$ (frequently, the input-to-state stability (ISS) framework $[10,11]$ is applied for this purpose). It implicitly implies that the error dynamics admits the following autonomous representation:

$$
\dot{e}(t)=\ell(e(t), d(t))
$$

for some $\ell: \mathbb{R}^{n+m} \rightarrow \mathbb{R}^{n}$, which is always the case for linear models (i.e. if $f(x, d)=A x+d$ and $h(x)=C x$ for some known matrices $A$ and $C$ of appropriate dimensions, then $r(z, y)=A z+L(y-C z), g(z)=z$ for a properly chosen gain $L$, and $\ell(e, d)=(A-L C) e+d)$, and often can also be obtained for the plants that are close to linear ones. However, in common scenario this differential equation has to take a more evolved form:

$$
\dot{e}(t)=\tilde{\ell}(e(t), x(t), d(t))
$$

with $\tilde{\ell}: \mathbb{R}^{2 n+m} \rightarrow \mathbb{R}^{n}$, then the estimation error dynamics has to be analyzed together with the observed system, and partial stability notions come to the attention, where one of the most popular concepts is studied in 
the input-to-output stability (IOS) theory [12, 13]. The latter situation (2) can also be analyzed using the quasiLPV methods [14], and it will be investigated here.

In this paper, the state estimation problem is studied for a nonlinear dynamical system that can be written in the Persidskii form, which has been widely investigated in the context of neural networks [15] and electric circuits [16]. Such a choice is justified since there is a known canonical form of Lyapunov function for a Persidskii system [17, 18, 19], and also by recent advancements in $[20,16]$ where both stability problems, in ISS and IOS sense, are constructively analyzed using linear matrix inequalities (LMIs). Hence, the stability for both cases of representation of the estimation error dynamics, in autonomous (1) and general settings (2), can be studied. For these two cases, the observer and corresponding stability conditions for a generalized Persidskii system are given in the sequel. Numerical experiments are presented to illustrate the performance of the designed observer.

The organization of this article is as follows. In Section 2 the preliminaries are presented. The system under consideration and the problem statement are described in Section 3. In sections 4 and 5 the LMI-based stability conditions for the cases (1) and (2) are given, respectively.

\section{Notation}

- $\mathbb{N}, \mathbb{R}$ and $\mathbb{R}_{+}$represent the sets of natural, real and nonnegative real numbers, respectively. The symbol $\|\cdot\|$ denotes the Euclidean norm on $\mathbb{R}^{n}$ (and the induced matrix norm $\|A\|$ for a matrix $A \in \mathbb{R}^{m \times n}$ ). The $n \times n$ diagonal matrix with $v \in \mathbb{R}^{n}$ on the main diagonal is denoted by $\operatorname{diag}(v)$. The set of diagonal matrices with nonnegative elements on the diagonal is denoted by $\mathbb{D}_{+}^{n} \subset \mathbb{R}_{+}^{n \times n}$.

- For $A \in \mathbb{R}^{n \times n}$ its maximal eigenvalue is denoted by $\lambda_{\max }(A)$.

- For $p, n \in \mathbb{N}$ with $p \leq n$, the notation $\overline{p, n}$ is used to represent the set of positive integers $\{p, \ldots, n\}$.

- For a Lebesgue measurable function $u: \mathbb{R}_{+} \rightarrow \mathbb{R}^{m}$, define the norm $\|u\|_{\left[t_{1}, t_{2}\right)}=\operatorname{ess} \sup _{t \in\left[t_{1}, t_{2}\right)}\|u(t)\|$ for $\left[t_{1}, t_{2}\right) \subset \mathbb{R}_{+}$. We denote by $\mathscr{L}_{\infty}^{m}$ the set of functions $u$ with $\|u\|_{\infty}:=\|u\|_{[0, \infty)}<+\infty$.

- A continuous function $\sigma: \mathbb{R}_{+} \rightarrow \mathbb{R}_{+}$belongs to class $\mathscr{K}$ if it is strictly increasing and $\sigma(0)=0$; it belongs to class $\mathscr{K}_{\infty}$ if it is also unbounded. A continuous function $\beta: \mathbb{R}_{+} \times \mathbb{R}_{+} \rightarrow \mathbb{R}_{+}$belongs to class $\mathscr{K} \mathscr{L}$ if $\beta(\cdot, r) \in \mathscr{K}$ and $\beta(r, \cdot)$ is a decreasing function going to zero for any fixed $r>0$.
- For a continuously differentiable function $V: \mathbb{R}^{n} \rightarrow \mathbb{R}$, denote by $\nabla V(p) v$ the derivative of $V$ at $p \in \mathbb{R}^{n}$ in the direction of $v \in \mathbb{R}^{n}$.

\section{Preliminaries}

Consider a nonlinear dynamical system:

$$
\begin{aligned}
& \dot{x}(t)=f(x(t), d(t)), t \in \mathbb{R}_{+}, \text {with } x(0)=x_{0}, \\
& y(t)=h(x(t)),
\end{aligned}
$$

where $x(t) \in \mathbb{R}^{n}$ is the state vector, $d(t) \in \mathbb{R}^{m}$ is the external perturbation, $d \in \mathscr{L}_{\infty}^{m}$ and $y(t) \in \mathbb{R}^{p}$ is the output vector. Moreover $f: \mathbb{R}^{n} \times \mathbb{R}^{m} \rightarrow \mathbb{R}^{n}, f(0,0)=0$ is a locally Lipschitz continuous function and $h: \mathbb{R}^{n} \rightarrow \mathbb{R}^{p}$ is a continuously differentiable function. For an initial state $x_{0} \in \mathbb{R}^{n}$ and $d \in \mathscr{L}_{\infty}^{m}$ we denote the corresponding solution of the system (3) by $x\left(t, x_{0}, d\right)$, and the corresponding output is $y\left(t, x_{0}, d\right)=h\left(x\left(t, x_{0}, d\right)\right)$.

The system (3) is called forward complete if for all $x_{0} \in \mathbb{R}^{n}$ and $d \in \mathscr{L}_{\infty}^{m}$, the solution $x\left(t, x_{0}, d\right)$ is uniquely defined for all $t \in \mathbb{R}_{+}$.

In the rest of the paper, to lighten the notation the time-dependency of variables might remain implicitly understood, for instance we will write $x$ for $x(t)$.

Definition 1. A forward complete system (3) is said to be practical input-to-output stable (pIOS) if there exist $\beta \in \mathscr{K} \mathscr{L}, \gamma \in \mathscr{K}$ and $c \in \mathbb{R}_{+}$such that

$$
\left\|y\left(t, x_{0}, d\right)\right\| \leq \beta\left(\left\|x_{0}\right\|, t\right)+\gamma\left(\|d\|_{\infty}\right)+c
$$

for all $t \in \mathbb{R}_{+}$and any $x_{0} \in \mathbb{R}^{n}$ and $d \in \mathscr{L}_{\infty}^{m}$. If $c=0$, the system is called input-to-output stable (IOS). In the special case when $h(x)=x$, the IOS property is called input-to-state stability (ISS).

Definition 2. A forward complete system (3) is said to be uniformly bounded-input-bounded-state stable (UBIBS) if there exists $\sigma \in \mathscr{K}$ such that

$$
\left\|x\left(t, x_{0}, d\right)\right\| \leq \max \left\{\sigma\left(\left\|x_{0}\right\|\right), \sigma\left(\|d\|_{\infty}\right)\right\}, \forall t \in \mathbb{R}_{+}
$$

for all $x_{0} \in \mathbb{R}^{n}$ and $d \in \mathscr{L}_{\infty}^{m}$.

Definition 3. For the system (3), a smooth Lyapunov function $V: \mathbb{R}^{n} \rightarrow \mathbb{R}_{+}$is:

1. an IOS-Lyapunov function if there exist $\alpha_{1}, \alpha_{2} \in$ $\mathscr{K}_{\infty}, \chi \in \mathscr{K}$ and $\alpha_{3} \in \mathscr{K} \mathscr{L}$ such that

$$
\begin{aligned}
& \qquad \alpha_{1}(\|h(x)\|) \leq V(x) \leq \alpha_{2}(\|x\|), \\
& V(x) \geq \chi(\|d\|) \Rightarrow \nabla V(x) f(x, d) \leq-\alpha_{3}(V(x),\|x\|) \\
& \text { for all } x \in \mathbb{R}^{n} \text { and } d \in \mathbb{R}^{m} \text {. }
\end{aligned}
$$


2. an ISS-Lyapunov function if there exist $\alpha_{1}, \alpha_{2} \in$ $\mathscr{K}_{\infty}, \chi \in \mathscr{K}$ and $\alpha_{3} \in \mathscr{K} \mathscr{L}$ such that

$$
\begin{gathered}
\alpha_{1}(\|x\|) \leq V(x) \leq \alpha_{2}(\|x\|), \\
\|x\| \geq \chi(\|d\|) \Rightarrow \nabla V(x) f(x, d) \leq-\alpha_{3}(\|x\|)
\end{gathered}
$$

for all $x \in \mathbb{R}^{n}$ and $d \in \mathbb{R}^{m}$.

Theorem 4 ([13]). A UBIBS system (3) is IOS if and only if it admits an IOS-Lyapunov function.

Theorem 5 ([13]). The system (3) is ISS if and only if it admits an ISS-Lyapunov function.

\section{Problem Statement}

Consider a class of systems in the Persidskii form:

$$
\begin{aligned}
& \dot{x}(t)=A_{0} x(t)+\sum_{j=1}^{M} A_{j} f^{j}(x(t))+D w(t), \\
& y(t)=\left[\begin{array}{c}
C_{0} x(t) \\
C_{1} f^{1}(x(t)) \\
\vdots \\
C_{M} f^{M}(x(t))
\end{array}\right]+v(t), t \in \mathbb{R}_{+},
\end{aligned}
$$

where $\quad x(t)=\left[\begin{array}{lll}x_{1}(t) & \ldots & x_{n}(t)\end{array}\right]^{\top} \in \mathbb{R}^{n}$ is the state vector; $A_{s} \in \mathbb{R}^{n \times n}$ for $s \in \overline{0, M}$; $f^{j}(x(t))=\left[f_{1}^{j}\left(x_{1}(t)\right) \ldots f_{n}^{j}\left(x_{n}(t)\right)\right]^{\top} \quad$ for $\quad j \in \overline{1, M}$ are the functions ensuring the existence of the solutions of the system (7) in the forward time at least locally; $y(t) \in \mathbb{R}^{z}$ is the output; $z=\sum_{s=0}^{M} z_{s}$ and $C_{s} \in \mathbb{R}^{z_{s} \times n}$ for $s \in \overline{0, M} ; \quad D \in \mathbb{R}^{n \times p} ; w(t) \in \mathbb{R}^{p}, v(t) \in \mathbb{R}^{z}$ are the external perturbations, $w \in \mathscr{L}_{\infty}^{p}, v \in \mathscr{L}_{\infty}^{z}$. The corresponding solution of the system (7) at time $t$ with initial state $x_{0} \in \mathbb{R}^{n}$ and disturbance $w \in \mathscr{L}_{\infty}^{p}$ is denoted by $x=x\left(t, x_{0}, w\right)$.

In this work, if for an index the upper bound is smaller than the lower one, then the corresponding term (in sum or a sequence) must be omitted.

The sector restrictions on $f^{j}, j \in \overline{1, M}$, are imposed as:

Assumption 1. Assume that for any $i \in \overline{1, n}$ and $j \in \overline{1, M}$,

$$
v f_{i}^{j}(v)>0, \forall v \in \mathbb{R} \backslash\{0\} .
$$

Under this assumption, with a reordering of nonlinearities and their decomposition, there exists an index $m \in \overline{0, M}$ such that for all $i \in \overline{1, n}, k \in \overline{1, m}$

$$
\lim _{v \rightarrow \pm \infty} f_{i}^{k}(v)= \pm \infty
$$

and that there exists $\mu \in \overline{m, M}$ such that for all $i \in \overline{1, n}$, $k \in \overline{1, \mu}$

$$
\lim _{v \rightarrow \pm \infty} \int_{0}^{v} f_{i}^{k}(\gamma) d \gamma=+\infty .
$$

In such a case $m=0$ implies that all nonlinearities are bounded (obviously $\mu \geq m$ ).

In this study, an observer for (7) is proposed in the following conventional form:

$$
\begin{aligned}
& \dot{\hat{x}}(t)=A_{0} \hat{x}(t)+\sum_{j=1}^{M} A_{j} f^{j}(\hat{x}(t))+L(y(t)-\hat{y}(t)), \\
& \hat{y}(t)=\left[\begin{array}{c}
C_{0} \hat{x}(t) \\
C_{1} f^{1}(\hat{x}(t)) \\
\vdots \\
C_{M} f^{M}(\hat{x}(t))
\end{array}\right],
\end{aligned}
$$

where $\hat{x}(t) \in \mathbb{R}^{n}$ is the estimation of the state $x(t) ; L_{s} \in$ $\mathbb{R}^{n \times z_{s}}$ for $s \in \overline{0, M}$ and $L=\left[L_{0} L_{1} \ldots L_{M}\right] \in \mathbb{R}^{n \times z}$ is the matrix gain to be designed.

Next for this observer we will analyze two cases of representation of the estimation error $e=x-\hat{x}$ given in introduction, (1) and (2). For the former case we will investigate IOS conditions for the common system (7), (8) with respect to the output error, while for the latter scenario, ISS conditions of the error dynamics of $e$ will be studied.

\section{IOS analysis}

The output stability for the system (7), (8) is equivalent to a robust state synchronization of these two Persidskii systems under the influence of perturbations $w$ and $v$. Thus, to apply the synchronization method developed in [16], let us represent the common dynamics of (7), (8) as

$$
\dot{X}=\tilde{A}_{0} X+\sum_{j=1}^{M} \tilde{A}_{j} F^{j}(X)+\mathscr{D},
$$

where $X=\left[x^{\top} \hat{x}^{\top}\right]^{\top} \in \mathbb{R}^{2 n}$ is the extended state and $\mathscr{D}:=\tilde{D}\left[w^{\top} v^{\top}\right]^{\top} \in \mathbb{R}^{2 n}$ is the augmented disturbance,

$$
\begin{gathered}
\tilde{A}_{s}=\left[\begin{array}{cc}
A_{s} & 0 \\
L_{s} C_{s} & A_{s}-L_{s} C_{s}
\end{array}\right], \forall s \in \overline{0, M} ; \\
F^{j}(X)=\left[\begin{array}{l}
f^{j}(x) \\
f^{j}(\hat{x})
\end{array}\right], \forall j \in \overline{1, M} ; \tilde{D}=\left[\begin{array}{cc}
D & 0 \\
0 & L
\end{array}\right]
\end{gathered}
$$

with the output function (the estimation or synchronization error):

$$
e=\Gamma X:=\left[\begin{array}{ll}
I_{n} & -I_{n}
\end{array}\right] X .
$$

It is clear that the system (9) also has the Persidskii form. Following [16], we say that if the two subsystems in the system (9) are robustly synchronized, then the estimation error $e$ satisfies the condition (4). In other words, (8) is an observer for (7) if the common dynamics (9) is IOS (this corresponds to the case (2) given 
in the introduction). The related conditions are as follows:

Theorem 6. Let Assumption 1 be satisfied. If there exist $0 \leq P_{1}=P_{1}^{\top} \in \mathbb{R}^{n \times n}, 0 \leq P_{2}=P_{2}^{\top} \in \mathbb{R}^{2 n \times 2 n}, \Lambda^{j}=$ $\operatorname{diag}\left(\Lambda_{1}^{j}, \ldots, \Lambda_{2 n}^{j}\right) \in \mathbb{D}_{+}^{2 n}$ for $j \in \overline{1, M}, \Theta \in \mathbb{D}_{+}^{2 n}, \Psi \in \mathbb{D}_{+}^{n}$, $\Xi^{k} \in \mathbb{D}_{+}^{2 n}$ for $k \in \overline{0, M}, \Upsilon_{s, z} \in \mathbb{D}_{+}^{2 n}$ for $s \in \overline{0, M-1}$, $z \in \overline{s+1, M}$ and $0<\Phi=\Phi^{\top} \in \mathbb{R}^{2 n \times 2 n}$ such that

$$
P_{1}>0 \text { or } P_{2}>0 \text { or } \sum_{j=1}^{\mu} \Lambda^{j}>0 ; P_{2} \leq \Theta, Q \leq 0,
$$

where

$$
\begin{gathered}
Q=\left[\begin{array}{cccccc}
Q_{1,1} & Q_{1,2} & Q_{1,3} & \cdots & Q_{1, M+1} & P_{\Gamma} \\
Q_{1,2}^{\top} & Q_{2,2} & Q_{2,3} & \cdots & Q_{2, M+1} & \Lambda^{1} \\
Q_{1,3}^{\top} & Q_{2,3}^{\top} & Q_{3,3} & \cdots & Q_{3, M+1} & \Lambda^{2} \\
\vdots & \vdots & \vdots & \ddots & \vdots & \vdots \\
Q_{1, M+1}^{\top} & Q_{2, M+1}^{\top} & Q_{3, M+1}^{\top} & \cdots & Q_{M+1, M+1} & \Lambda^{M} \\
P_{\Gamma} & \Lambda^{1} & \Lambda^{2} & \cdots & \Lambda^{M} & -\Phi
\end{array}\right], \\
P_{\Gamma}=\Gamma^{\top} P_{1} \Gamma+P_{2} ; Q_{1,1}=\tilde{A}_{0}^{\top} P_{\Gamma}+P_{\Gamma} \tilde{A}_{0}+\Xi^{0}+\Gamma^{\top} \Psi \Gamma, \\
Q_{j+1, j+1}=\tilde{A}_{j}^{\top} \Lambda^{j}+\Lambda^{j} \tilde{A}_{j}+\Xi^{j}, j \in \overline{1, M}, \\
Q_{1, j+1}=P_{\Gamma} \tilde{A}_{j}+\tilde{A}_{0}^{\top} \Lambda^{j}+\Upsilon_{0, j}, j \in \overline{1, M}, \\
Q_{s+1, z+1}=\tilde{A}_{s}^{\top} \Lambda^{z}+\Lambda^{s} \tilde{A}_{z}+\Upsilon_{s, z}, s \in \overline{1, M-1}, z \in \overline{s+1, M},
\end{gathered}
$$

then a forward complete system (9) is IOS if

$$
P_{1} \leq \xi \Psi, \Theta+\sum_{j=1}^{M} \Lambda^{j} \leq \xi\left(\sum_{k=0}^{m} \Xi^{k}+2 \sum_{s=0}^{m-1} \sum_{z=s+1}^{m} \Upsilon_{s, z}\right)
$$

for some $\xi>0$.

Proof. Consider a candidate Lyapunov function

$$
V(X)=X^{\top} P_{\Gamma} X+2 \sum_{j=1}^{M} \sum_{i=1}^{2 n} \Lambda_{i}^{j} \int_{0}^{X_{i}^{j}} F_{i}^{j}(\tau) d \tau .
$$

If $P_{1}>0$, then

$$
e^{\top} P_{1} e \leq V(X) \leq \alpha_{2}(\|X\|),
$$

with

$\alpha_{2}(\tau) \leq \lambda_{\max }\left(P_{\Gamma}\right) \tau^{2}+2 n M \max _{i \in 1,2 n, j \in 1, M}\left\{\Lambda_{i}^{j} \int_{0}^{\tau} F_{i}^{j}(\gamma) d \gamma\right\}$

a function from class $\mathscr{K}_{\infty}$, so the first condition in (5) is verified. Next, consider the derivative of $V$ :

$$
\begin{aligned}
\dot{V}= & {\left[\begin{array}{c}
X \\
F^{1}(X) \\
\vdots \\
F^{M}(X) \\
\mathscr{D}
\end{array}\right]^{\top} Q\left[\begin{array}{c}
X \\
F^{1}(X) \\
\vdots \\
F^{M}(X) \\
\mathscr{D}
\end{array}\right]-X^{\top}\left(\Gamma^{\top} \Psi \Gamma+\Xi^{0}\right) X } \\
& -\sum_{j=1}^{M} F^{j}(X)^{\top} \Xi^{j} F^{j}(X)-2 \sum_{j=1}^{M} X^{\top} \Upsilon_{0, j} F^{j}(X) \\
& -2 \sum_{s=1}^{M-1} \sum_{z=s+1}^{M} F^{s}(X)^{\top} \Upsilon_{s, z} F^{z}(X)+\mathscr{D}^{\top} \Phi \mathscr{D}
\end{aligned}
$$

under (10). Due to the form of the function $V$, there exists $\alpha \in \mathscr{K}_{\infty}$ such that

$$
\begin{aligned}
& \alpha(V(X)) \leq X^{\top}\left(\Gamma^{\top} \Psi \Gamma+\Xi^{0}\right) X+\sum_{j=1}^{M} F^{j}(X)^{\top} \Xi^{j} F^{j}(X) \\
& +2 \sum_{j=1}^{M} X^{\top} \Upsilon_{0, j} F^{j}(X)+2 \sum_{s=1}^{M-1} \sum_{z=s+1}^{M} F^{s}(X)^{\top} \Upsilon_{s, z} F^{z}(X)
\end{aligned}
$$

under the conditions (11), which have to be verified for some $\xi>0$ (only the first $m$ nonlinearities and the quadratic term are radially unbounded). Therefore, under the conditions of the theorem: $\dot{V} \leq-\alpha(V)+\mathscr{D}^{\top} \Phi \mathscr{D}$ for all $X \in \mathbb{R}^{2 n}$ and $\mathscr{D} \in \mathbb{R}^{2 n}$. If $\bar{V} \geq \alpha^{-1}\left(2 \mathscr{D}^{\top} \Phi \mathscr{D}\right)$, then the second relation in (5) can be recovered

$$
V \geq \alpha^{-1}\left(2 \mathscr{D}^{\top} \Phi \mathscr{D}\right) \Rightarrow \dot{V} \leq-\frac{1}{2} \alpha(V),
$$

and the IOS property is guaranteed (if the right-hand side of the estimate for $\dot{V}$ is in the form of a function of class $\mathscr{K}$, as above and not of class $\mathscr{K} \mathscr{L}$ as in (5), then UBIBS property can be omitted, and a forward completeness is enough).

The proofs of the theorems and corollary in the sequel are skipped due to space limitations.

We can require more strict properties from the nonlinearities of the system (7):

Assumption 2. For any $j \in \overline{1, M}$ :

$X^{\top} \Gamma^{\top} \Gamma F^{j}(X)>0, \quad \forall X \in \mathbb{R}^{2 n} \backslash\left\{X \in \mathbb{R}^{2 n}: \Gamma X=0\right\}$.

Under these additional restrictions imposed on the system (9), a relaxed stability result can be obtained:

Corollary 7. Let the assumptions 1 and 2 be satisfied. If there exist $0 \leq P_{1}=P_{1}^{\top} \in \mathbb{R}^{n \times n}, 0 \leq P_{2}=P_{2}^{\top} \in$ $\mathbb{R}^{2 n \times 2 n} ; \Xi^{k} \in \mathbb{D}_{+}^{n}$ for $k \in \overline{0, M} ; \Lambda^{j} \in \mathbb{D}_{+}^{2 n}$ for $j \in \overline{1, M} ;$ $\Upsilon_{s, z} \in \mathbb{D}_{+}^{n}$ for $s \in \overline{0, M-1}$ and $z \in \overline{s+1, M}$ such that

$$
P_{2}>0 \text { or } \sum_{q=1}^{\mu} \Lambda^{q}>0 ; Q \leq 0
$$

where

$$
\begin{gathered}
Q=\left\{Q_{k k^{\prime}}\right\}_{k, k^{\prime}=1}^{M+1}, P_{\Gamma}=\Gamma^{\top} P_{1} \Gamma+P_{2} ; Q_{1,1}=\tilde{A}_{0}^{\top} P_{\Gamma}+P_{\Gamma} \tilde{A}_{0}+\Gamma^{\top} \Xi^{0} \Gamma, \\
Q_{j+1, j+1}=\tilde{A}_{j}^{\top} \Lambda^{j}+\Lambda^{j} \tilde{A}_{j}+\Gamma^{\top} \Xi^{j} \Gamma, j \in \overline{1, M}, \\
Q_{1, j+1}=P_{\Gamma} \tilde{A}_{j}+\tilde{A}_{0}^{\top} \Lambda^{j}+\Gamma^{\top} \Upsilon_{0, j} \Gamma, j \in \overline{1, M}, \\
Q_{s+1, z+1}=\tilde{A}_{s}^{\top} \Lambda^{z}+\Lambda^{s} \tilde{A}_{z}+\Gamma^{\top} \Upsilon_{s, z} \Gamma, s \in \overline{1, M-1}, z \in \overline{s+1, M} .
\end{gathered}
$$

Then the system (9) with $\mathscr{D}(t)=0$ for all $t \in \mathbb{R}_{+}$has globally bounded trajectories and $\lim _{t \rightarrow+\infty}\|e(t)\|=0$ if

$$
\sum_{k=0}^{M} \Xi^{k}+2 \sum_{s=0}^{M-1} \sum_{z=s+1}^{M} \Upsilon_{s, z}>0
$$


Example 8. In this example the system

$$
\dot{X}=\tilde{A}_{0} X+\tilde{A}_{1} F^{1}(X)
$$

in the form of (9) with $w(t)=0, v(t)=0$ for all $t \in$ $\mathbb{R}_{+}$is considered, where $x=\left[\begin{array}{ll}x_{1} & x_{2}\end{array}\right]^{\top}, \hat{x} \in \mathbb{R}^{2}$ are the states,

$$
\begin{gathered}
A_{0}=\left[\begin{array}{ll}
0.0849 & -0.2145 \\
0.2488 & -0.2266
\end{array}\right], A_{1}=\left[\begin{array}{cc}
-0.7030 & -0.7541 \\
0.5473 & -0.5535
\end{array}\right], \\
L_{0}=\left[\begin{array}{l}
0.6306 \\
0.9855
\end{array}\right], L_{1}=\left[\begin{array}{l}
0.6343 \\
0.6005
\end{array}\right], F^{1}(X):=\left[\begin{array}{l}
f^{1}(x) \\
f^{1}(\hat{x})
\end{array}\right], \\
C_{1}=\left[\begin{array}{l}
0.3354 \\
0.9571
\end{array}\right]^{\top}, f^{1}(x):=\left[\begin{array}{l}
\frac{x_{1}}{\left|x_{1}\right|+1} \\
\left|x_{2}\right|+2
\end{array}\right], C_{0}=\left[\begin{array}{ll}
-1 & 2
\end{array}\right] .
\end{gathered}
$$

Then the LMIs proposed in Corollary 7 are verified. The states of the systems (7), (8) with two distinct initial conditions $x(0), \hat{x}(0)$ and their errors are shown in Fig. 1 and Fig. 2, respectively (the time and the error norm in Fig. 2 are shown in logarithmic scale). The simulation results indicate that the behavior of the system (7) (it has nonlinearities in the state and in the output equations) is well estimated by the observer (8).
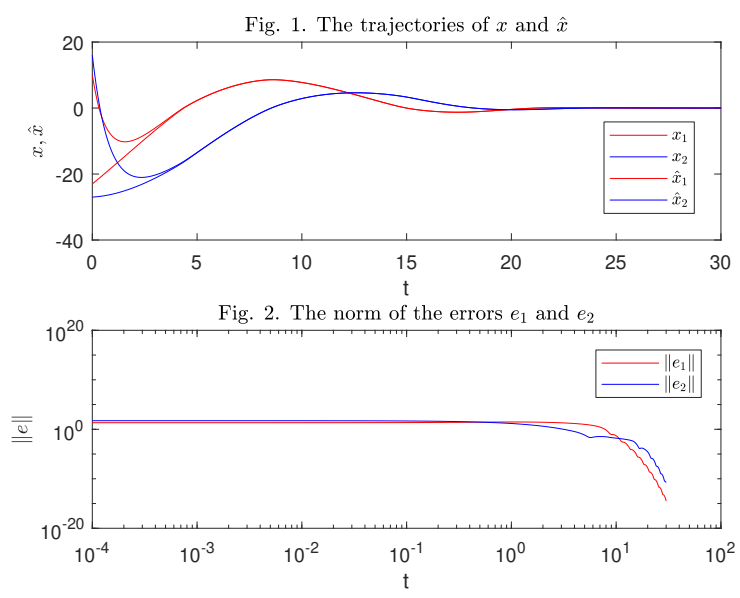

\section{ISS analysis}

Let us introduce a short-hand notation $\delta f^{j}:=$ $f^{j}(x)-f^{j}(\hat{x})$ for all $j \in \overline{1, M}$ (by skipping the arguments $(x, \hat{x})$ ). Then the following properties are required in the sequel for nonlinear functions in the system (7):

Assumption 3. There exist $S_{0}^{j}, S_{1}^{j}, S_{2}^{j}, S_{3}^{j, k} \in \mathbb{D}_{+}^{n}$ with $j, k \in \overline{1, M}$ such that

$$
\begin{aligned}
\left(\delta f^{j}\right)^{\top} \delta f^{j} \leq & e^{\top} S_{0}^{j} e+2 e^{\top} S_{1}^{j}\left(\delta f^{j}\right)+2 e^{\top} S_{2}^{j} f^{j}(e) \\
& +2 \sum_{k=1}^{M}\left(\delta f^{j}\right)^{\top} S_{3}^{j, k} f^{k}(e)
\end{aligned}
$$

for all $x, \hat{x} \in \mathbb{R}^{n}$ with $e:=x-\hat{x}$.
Applying the observer (8) to the system (7), the estimation error dynamics $e$ can be rewritten as:

$$
\dot{e}=\mathscr{A}_{0} e+\sum_{j=1}^{M} \mathscr{A}_{j} \delta f^{j}+\mathscr{D},
$$

where $\mathscr{D}:=L v+D w$ is another auxiliary bounded input and $\mathscr{A}_{s}=A_{s}-L_{s} C_{s}, \forall s \in \overline{0, M}$. Under Assumption 3 the error dynamics (15) can be interpreted as the autonomous system (1).

Theorem 9. Let the assumptions 1,2 and 3 be satisfied. If there exist $0 \leq P=P^{\top} \in \mathbb{R}^{n \times n}, \Lambda^{j}=$ $\operatorname{diag}\left(\Lambda_{1}^{j}, \ldots, \Lambda_{n}^{j}\right), \Gamma_{j}, \Omega_{j} \in \mathbb{D}_{+}^{n}$ for $j \in \overline{1, M}, \Xi^{0} \in \mathbb{D}_{+}^{n}$, $\Upsilon_{j, k} \in \mathbb{D}_{+}^{n}$ for $j, k \in \overline{1, M}, 0<\Phi=\Phi^{\top} \in \mathbb{R}^{n \times n}$ and $\gamma>0$ such that

$$
\begin{gathered}
P>0 \text { or } \sum_{j=1}^{\mu} \Lambda^{j}>0 ; \mathscr{Q} \leq 0 \\
\Xi^{0}-\gamma \sum_{j=1}^{M} S_{0}^{j} \geq 0, \Gamma_{j}-\gamma S_{1}^{j} \geq 0, \Omega_{j}-\gamma S_{2}^{j} \geq 0, \\
\Upsilon_{j, k}-\gamma S_{3}^{j, k} \geq 0, \Xi^{0}-\sum_{j=1}^{M} \gamma S_{0}^{j}+\sum_{j=1}^{m}\left(\Omega_{j}-\gamma S_{2}^{j}\right)>0,
\end{gathered}
$$

where

$$
\begin{aligned}
& \mathscr{Q}=\left[\begin{array}{cccc}
\mathscr{Q}_{1,1} & \mathscr{Q}_{1,2} & \mathscr{Q}_{1,3} & P \\
\mathscr{Q}_{1,2}^{\top} & \mathscr{2}_{2,2} & \mathscr{Q}_{2,3} & 0 \\
\mathscr{Q}_{1,3}^{\top} & \mathscr{Q}_{2,3}^{\top} & 0 & \mathscr{Q}_{3,4} \\
P & 0 & \mathcal{Q}_{3,4}^{\top} & -\Phi
\end{array}\right] ; \\
& \mathscr{Q}_{1,1}=\mathscr{A}_{0}^{\top} P+P \mathscr{A}_{0}+\Xi^{0}, \mathscr{Q}_{2,2}=-\operatorname{diag}\left(\gamma I_{n}, \ldots, \gamma I_{n}\right), \\
& \mathscr{Q}_{1,2}=\left[\begin{array}{lll}
P \mathscr{A}_{1}+\Gamma_{1} & \ldots & P \mathscr{A}_{M}+\Gamma_{M}
\end{array}\right], \\
& \mathscr{Q}_{1,3}=\left[\begin{array}{lll}
\mathscr{A}_{0}^{\top} \Lambda^{1}+\Omega_{1} & \ldots & \mathscr{A}_{0}^{\top} \Lambda^{M}+\Omega_{M}
\end{array}\right], \\
& \mathscr{Q}_{3,4}=\left[\begin{array}{lll}
\Lambda^{1} & \ldots & \Lambda^{M}
\end{array}\right]^{\top}, \\
& \mathscr{Q}_{2,3}=\left[\begin{array}{ccc}
\mathscr{A}_{1}^{\top} \Lambda^{1}+\Upsilon_{1,1} & \cdots & \mathscr{A}_{1}^{\top} \Lambda^{M}+\Upsilon_{1, M} \\
\vdots & \ddots & \vdots \\
\mathscr{A}_{M}^{\top} \Lambda^{1}+\Upsilon_{M, 1} & \cdots & \mathscr{A}_{M}^{\top} \Lambda^{M}+\Upsilon_{M, M}
\end{array}\right],
\end{aligned}
$$

then the estimation error dynamics (15) is ISS.

Remark 10. Under an assumption that there exists $\alpha \in$ $\mathscr{K}_{\infty}$ such that

$$
\left(\delta f^{j}\right)^{\top} \delta f^{j} \geq \alpha(\|e\|)
$$

for all $x, \hat{x} \in \mathbb{R}^{n}$ and all $j \in \overline{1, M}$ (i.e., the functions $\delta f^{j}$ are radially unbounded in terms of the estimation error $e$ ), the conditions (16) can be relaxed as:

$$
\begin{gathered}
P>0 \text { or } \sum_{j=1}^{\mu} \Lambda^{j}>0 ; \mathscr{Q} \leq 0 ; \Xi^{0}-\gamma \sum_{j=1}^{M} S_{0}^{j} \geq 0, \Gamma_{j}-\gamma S_{1}^{j} \geq 0, \\
\Omega_{j}-\gamma S_{2}^{j} \geq 0, \Upsilon_{j, k}-\gamma S_{3}^{j, k} \geq 0 ; \Xi^{0}-\gamma \sum_{j=1}^{M} S_{0}^{j}+2 \sum_{j=1}^{M}\left(\Gamma_{j}-\gamma S_{1}^{j}\right) \\
\quad+2 \sum_{j=1}^{M}\left(\Omega_{j}-\gamma S_{2}^{j}\right)+2 \sum_{j=1}^{M} \sum_{k=1}^{M}\left(\Upsilon_{j, k}-\gamma S_{3}^{j, k}\right)>0 .
\end{gathered}
$$


Example 11. Consider Example 8. Let additionally $w(t)=\sin (3 t), v(t)=[\sin (3 t) \sin (5 t)]^{\top}$ and $D=\left[\begin{array}{ll}1 & 3\end{array}\right]^{\top}$ for $t \in \mathbb{R}_{+}$. Assumption 3 is satisfied for

$$
\begin{gathered}
S_{0}^{1}=\operatorname{diag}(0.0138,0.0489), S_{1}^{1}=\operatorname{diag}(0.4014,0.6028), \\
S_{2}^{1}=10^{-4} \times \operatorname{diag}(0.0761,0.1397), \\
S_{3}^{1,1}=10^{-4} \times \operatorname{diag}(0.4990,0.6582) .
\end{gathered}
$$

Using the estimation error dynamics representation (15), we obtain that the LMIs of Theorem 9 are verified. The corresponding error trajectories in logarithmic time scale are shown in Fig. 3, where the errors are converging to a vicinity of the origin proportional to the amplitude of disturbances.

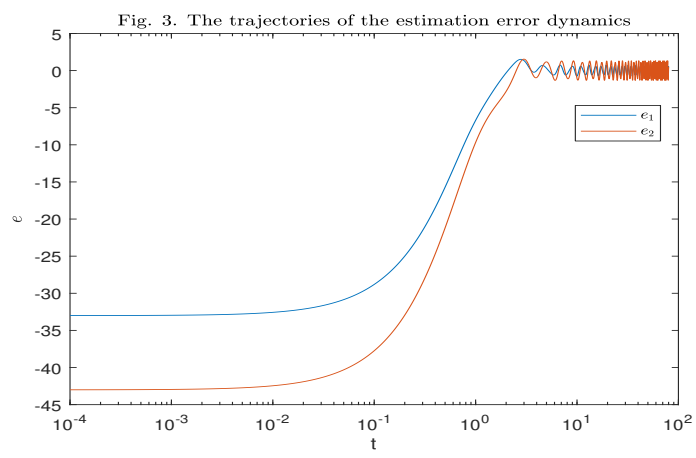

\section{Conclusion}

In this work, the problem of state estimation for a class of Persidskii systems was studied. A simple observer was proposed containing a copy of the system dynamics with an output injection term. Two sets of stability conditions were developed: establishing IOS of the common dynamics of the system and the observer with respect to the estimation error, and providing ISS of the estimation error dynamics. These conditions were obtained in the form of LMIs. Two academic examples were shown to verify the effectiveness of the introduced framework.

\section{References}

[1] H. Khalil, Nonlinear systems. Upper Saddle River, NJ: Prentice-Hall, 2002.

[2] G. Besançon, Nonlinear observers and applications, ser. Lecture notes in control and information. Springer, 2007, vol. 363.

[3] R. Kalman, "A new approach to linear filtering and prediction problems," Journal of Basic Engineering, vol. 82, pp. 35-45, 1960.

[4] D. Luenberger, "An introduction to observers," IEEE Transactions on Automatic Control, vol. 16, no. 6, pp. 596-602, 1971.
[5] A. J. Krener and W. Respondek, "Nonlinear observers with linearizable error dynamics," SIAM Journal on Control and Optimization, vol. 23, no. 2, pp. 197-216, 1985.

[6] H. Hammouri and M. Kinnaert, "A new procedure for time-varying linearization up to output injection," $S y$ stems \& Control Letters, vol. 28, no. 3, pp. 151-157, 1996.

[7] S. Särkkä, "On unscented Kalman filtering for state estimation of continuous-time nonlinear systems," IEEE Transactions on Automatic Control, vol. 52, pp. 16311641, 2007.

[8] R. Kandepu, B. Foss, and L. Imsland, "Applying the unscented Kalman filter for nonlinear state estimation," Journal of Process Control, vol. 18, pp. 753-768, 2008.

[9] C. Rao, J. Rawlings, and D. Mayne, "Constrained state estimation for nonlinear discrete-time systems: stability and moving horizon approximations," IEEE Transactions on Automatic Control, vol. 48, pp. 246-258, 2003.

[10] E. D. Sontag, "The ISS philosophy as a unifying framework for stability-like behavior," in Nonlinear control in the year 2000, Vol. 2 (Paris), ser. Lecture Notes in Control and Inform. Sci. London: Springer, 2001, vol. 259, pp. 443-467.

[11] S. Dashkovskiy, D. Efimov, and E. Sontag, "Input to state stability and allied system properties," Automation and Remote Control, vol. 72, no. 8, pp. 1579-1614, 2011.

[12] E. D. Sontag and Y. Wang, "Notions of input to output stability," Systems \& Control Letters, vol. 38, no. 4-5, pp. 235-248, 1999.

[13] E. Sontag and Y. Wang, "Lyapunov characterizations of input to output stability," SIAM Journal on Control and Optimization, vol. 39, no. 1, pp. 226-249, 2000.

[14] F.-R. López-Estrada, D. Rotondo, and G. ValenciaPalomo, "A review of convex approaches for control, observation and safety of linear parameter varying and Takagi-Sugeno systems," Processes, vol. 7, no. 11, p. $814,2019$.

[15] J. Hopfield and D. Tank, "Computing with neural circuits: a model," Science, vol. 233 , no. 4764 , pp. $625-$ 633, 1986.

[16] W. Mei, D. Efimov, and R. Ushirobira, "Feedback synchronization in Persidskii systems," Proc. 21st IFAC World Congress, 2020.

[17] E. Barbashin, "On construction of Lyapunov functions for nonlinear systems," in Proc. 1st IFAC World Congress, Moscow, 1961, pp. 742-751.

[18] S. Persidskii, "Concerning problem of absolute stability," Automation and Remote Control, pp. 5-11, 1969.

[19] E. Kaszkurewicz and H. Liu, "Stability of nonlinear systems," Automatica, vol. 15, pp. 609-614, 1979.

[20] D. Efimov and A. Aleksandrov, "Robust stability analysis and implementation of Persidskii systems," 58th IEEE Conference on Decision and Control (CDC), 2019. 